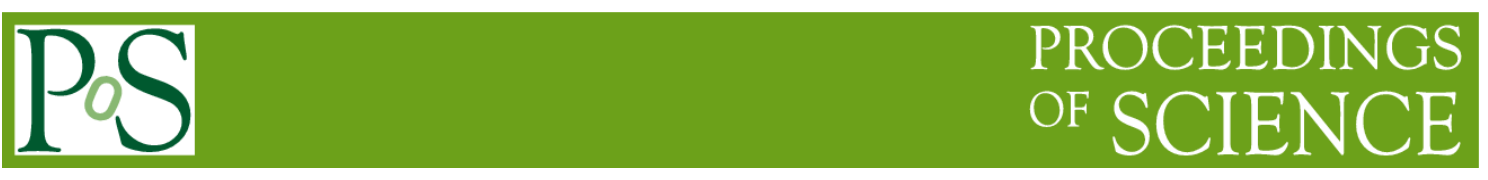

\title{
GPS/INS Integrated Navigation System Suitable for Indoor and Outdoor Positioning
}

\author{
Heding Shi ${ }^{1}$ \\ Shanghai University \\ Shanghai, 200072, China \\ E-mail: shdxs19900163.com
}

\section{Tongyue Gao}

Shanghai University

Shanghai, 200072, China

E-mail: gtyeshu. edu. cn

\section{Haifeng Liu}

Shanghai University

Shanghai, 200072, China

E-mail: LiuHaiFeng_Arvin@163.com

In an open sky environment, GPS is able to provide the navigation information with relatively high precision, including position, speed and direction. However, in complex urban canyons or indoors, the validity of GPS information is restricted and it can't provide accurate results or even fails in use. In this paper, an integrated navigation system for the pedestrian is introduced, which is based on physiological features of the pedestrian's gait through step detection and step length estimation. With the transformation of the environment, specific strategy is adopted. This solution can maintain the accuracy, reliability and continuity of positioning in any environment. The paper mainly introduces how to obtain an accurate digital platform for the integrated navigation system and use the platform to estimate the velocity and heading for pedestrians to implement the seamless positioning and navigation.

ISCC 2015

18-19, December, 2015

Guangzhou, China

\footnotetext{
${ }^{1}$ Speaker/Correspongding Author
} 


\section{Introduction}

With constant breakthroughs of mobile communications technology, continual expansion of the intelligent mobile terminal and rapid development of the positioning technology, application of the location-based service plays a more and more important role in people's daily life. The navigation system is useful for emergency responders, security personnel, and wide range augmented reality applications [1]. The central issue of these applications is how to obtain the position, velocity and heading information with a specified accuracy [2]. Global Positioning System (GPS) which is one of the most important positioning means can provide accurate location, speed and high precision time standards for the vast majority of the earth's surface area. Since pedestrian navigation systems are often used in such challenging environment as urban canyons or indoors, the use of GPS signals is often restricted [3]. Compared with GPS, the inertial measurement has the advantage of portablility, small quantization error and pricecompetitiveness [4]. However, its positioning precision must be accumulated over time. It is obvious that the technology composed of GPS and the inertial navigation can achieve the transition between the indoor and outdoor environment and ensure the availability of positioning information.

According to the position where sensors are stalled, Inertial Navigation System(INS) can be categorized into foot-mounted, waist-mounted and handheld types [5]. Moafipoor proposed a personal system installed in a backpack configuration, which relies on integrating knowledgebased systems and artificial intelligence methodologies. Although the accuracy of the system is $\pm(3-5) \mathrm{m}$ CEP50, it is too complex and difficult to use in practice [2]. Ojeta and Borenstein designed a six-axes inertial measurement unit attached to the user's boot and applied zero velocity update (ZUPT) for effectively minimizing the drift [6]. Zhang et al. introduced a handheld inertial pedestrian navigation system based on low-cost microelectromechanical system sensors. They developed a multiple classifier to recognize human step modes and device poses so that its accuracy can be improved [5].

In this paper, the system is bounded to the left side of the waist and regarded as the bodyfixed system. This system uses self-contained sensors to gain several measurements such as the acceleration, angular rate, temperature and altitude. Used in combination with the pedestrians' dead reckoning algorithm and the pedestrian kinematics model, Extended Kalman Filter(EKF) is designed to get the accurate speed and heading. However, the temperature fluctuation can affect sensor properties to such great extent that it can influence the performance of the system. What's worse, the pedestrian's posture change will lead to the error of the body coordinate system and bias of orientation. This paper mainly introduces: 1. how to obtain the accurate attitude in different environments and establish the accurate digital platform; 2 . how to fuse the information from GPS and INS to obtain the optimal value.

\section{System Description}

\subsection{Inertial Measurement Unit(IMU)}

In this paper, we design a gait navigation reference system, combining with the relative measurement method and pedestrian dead-reckoning model, which can obtain accurate, reliable 
and continuous information about the position, direction and speed, as shown in Fig. 1. This navigation system includes a three-axes accelerometer, a three-axes gyroscope, a temperature sensor and a barometer, and uses the Ublox NEO-M8 module to receive GPS messages and the Bluetooth module to contact PC or mobile phones. IMU gets all kinds of data periodically. Sample rates of raw data from the sensor are: $50 \mathrm{~Hz}$ for acceleration and gyroscope, $10 \mathrm{~Hz}$ for barometer and the temperature sensor, and $2 \mathrm{~Hz}$ for the GPS module. The key of this system lies in the obtainable accuracy and long term stability due to drifts of inertial sensors [7]. In addition, the accuracy of heading and velocity is improved by compensating several parameters, such as the installation error and oscillation of the human body when walking.

\subsection{Integrated Navigation}

The quality of signals of GPS messages is diverse on the basis of differences in environment. The best method consists in taking into account the characteristic that the GPS signal can export a high precision positioning result occasionally and positioning results from INS are accurate in a short time. EKF can export accurate digital platform and optimal results from the integration of GPS and INS positioning information, as shown in Fig. 2. According to the superiority of GPS signals, this dissertation proposes a three-mode positioning mechanism: when the GPS signal performs well, positioning results derive from the GPS receiver directly; when GPS messages can be obtained but not reliable, positioning results derive from outputs of the kalman filter of GPS and INS; when GPS signals are not useful, positioning results derive from the inertial navigation directly.

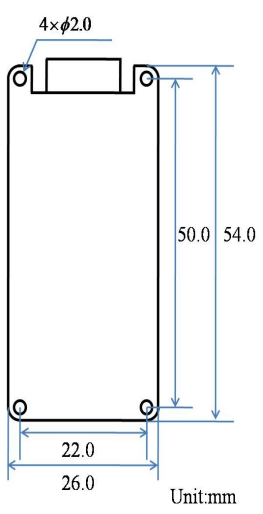

Figure 1: Gait Navigation Reference System

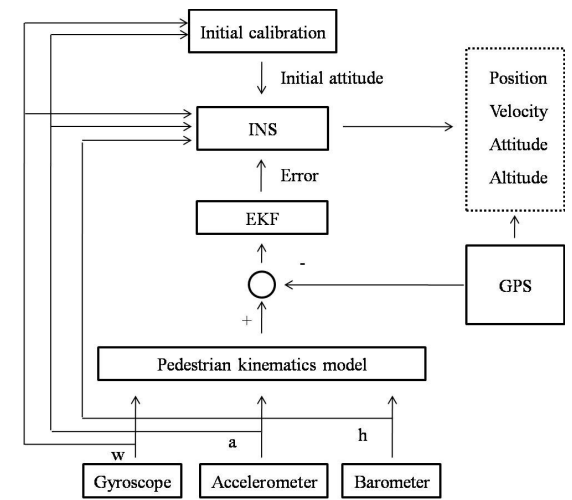

Figure 2: Integrated Navigation Framework

\section{Pedestrian Kinematics Model}

Variations in step modes and device poses seriously limit the performance of deadreckoning [5]. In pedestrian navigation systems, dead-reckoning is a popular method for estimating current position by using the number of steps taken, stride length and orientation incrementally [8]. We can get step length estimation and step detection through the three-axes acceleration. In a three-dimensional indoor environment, step modes of the pedestrian can be categorized into walking, making turns, in a static state, moving upstairs or moving downstairs [5]. 


\subsection{Step Detection}

In terms of the pedestrian navigation, it is important to know when the subject takes a step [9]. In order to keep body balance, everyone's normal walking gait is periodical. Each gait period includes four phases typically: feet off the ground, swinging, touching, and standing up. Three-axes acceleration measurements should be in the form of a cyclical change and reflect the occurrence. The accelerometer attached to the user's body can sense the movement by measuring the ground reaction force of the feet during walking. Step is detected by researching the maximum and minimum value of acceleration in every cycle. The number of steps is estimated by counting the number of peaks of the filter acceleration [10]. The situation of multiple-peak featuring oscillation of the pedestrian's body can be avoided effectively by gravity compensation and smooth filtering, as shown in Fig. 3.

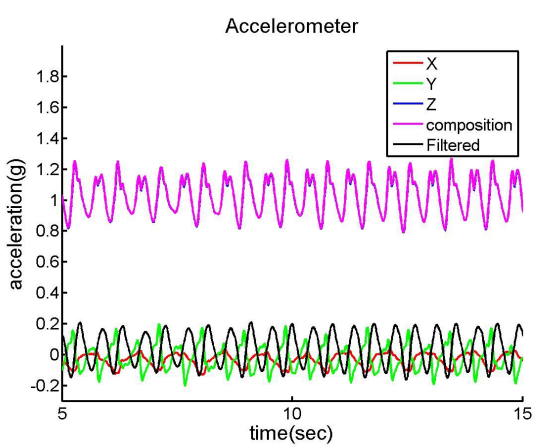

Figure 3: Three-axes Acceleration Measurements

\subsection{Step Length Estimation}

Human locomotion activity is a relatively complex phenomenon, generally characterized by its frequency, duration, intensity, environment, and the activity type [2]. Considering the body locomotion pattern and individual differences, there are several step models determining the step length in general: constant model, linear model, non-linear model, and so on. Based on characteristics of the body dynamic model, step length is mainly related to acceleration and frequency. Therefore, in this article, the following model is used to estimate step length:

$$
L=k \sqrt[b]{a_{\max }-a_{\min }}+\beta
$$

where $a_{\max }, a_{\min }$ denotes the maximum, minimum of filtered acceleration in the step cycle, $\quad b$ denotes the scale factor of step length, $\beta$ denotes the offset.

\subsection{State Assessment}

According to the pedestrian gait periodicity in walking, the frequency detection and step length estimation can be achieved effectively through using acceleration measurements in real time. The state determination includes the following conditions:

(1) Determining the occurrence of every step based on the accelerometer. The $Z$ axis acceleration peak value has to satisfy a certain condition. The maximum value must be larger than a threshold and the minimum value must be smaller than another threshold. When the acceleration variance is considered, results can be slightly improved [11]. This avoids errors caused by the vibration and the body shaking because of the randomness of signals. 


$$
C_{1}= \begin{cases}1 & a_{i b k \max }>t h_{\text {amax }} \& a_{i b k m i n}<t h_{\text {amin }} \\ 0 & \text { otherwise }\end{cases}
$$

(2) Determining the occurrence of every step based on the time interval. The frequency of normal walking is about $1-4 \mathrm{~Hz}$, so the time interval between the two similar peaks in successive periods must maintain in a reasonable range.

$$
C_{2}= \begin{cases}1 & t_{\text {tmin }}<|\Delta t|<t h_{\text {tmax }} \\ 0 & \text { otherwise }\end{cases}
$$

(3) Determining altitude variation based on the barometer. The altitude changes when we go downstairs or upstairs. Measurement from the barometer can reflect the present press value which is used to calculate the altitude directly. So the difference value of the vertical dimension can be obtained by the relative measuring method after smooth filtering. The value must stay in a reasonable range and the height must be known in advance.

$$
C_{3}=\left\{\begin{array}{cl}
1 & \Delta A>t h_{\text {Amax }} \\
0 & \text { otherwise } \\
-1 & \Delta A<t h_{\text {Amin }}
\end{array}\right.
$$

(4) Confirming the occurrence of the turn from the gyroscope. The $\mathrm{Z}$ axis angular rate value must be larger than a certain threshold when turning to normal walking.

$$
C_{4}= \begin{cases}1 & w_{i b}>t h_{w m i n} \\ 0 & \text { otherwise }\end{cases}
$$

Through the assessment of $C_{1}, C_{2}, C_{3}$ and $C_{4}$, we can confirm pedestrian motion modes, as shown in Table 1.

\begin{tabular}{|c|c|c|c|c|}
\hline$C_{1}$ & $C_{2}$ & $C_{3}$ & $C_{4}$ & Mode \\
\hline 1 & 1 & 1 & $/$ & go upstairs \\
\hline 1 & 1 & -1 & $/$ & go downstairs \\
\hline 1 & 1 & 0 & 0 & go straight \\
\hline 1 & 1 & 0 & 1 & turn \\
\hline 0 & 0 & $/$ & $/$ & \multirow{2}{*}{ in a static state } \\
\hline \multicolumn{2}{|c|}{ otherwise } \\
\hline
\end{tabular}

Table 1: State Assessment of the pedestrian

\section{Integrated Navigation}

In this paper, the navigation system is based on an accurate digital platform through initial calibration and attitude compensation. Combined with sport physiological features of the pedestrian gait, we use a three-axes acceleration sensor to detect frequency of the pedestrian movement and a three-axes angular rate sensor to obtain pedestrians' orientation information. The EKF algorithm is applied and the relative position is calculated in a navigation coordinate. If GPS provides the starting position for dead reckoning, it is easy to obtain the absolute position in ENU.

\subsection{Initial Calibration}

Sensors installed in the system are calibrated according to static and dynamic calibration processes[2]. The purpose of the initial alignment is to obtain the accurate posture in the initial 
moment. During the initial Calibration process, the body keeps static. The accelerometer measurement is the projection of the gravitational acceleration $(\mathrm{g})$ in the body coordinate $a^{b}=\left[\begin{array}{lll}a_{x} & a_{y} & a_{z}\end{array}\right]$ and the gyroscope measurement is the projection of the earth rotation angular velocity $\left(w_{i e}\right)$ in the body coordinate $w_{i e}^{b}=\left[\begin{array}{lll}w_{x} & w_{y} & w_{z}\end{array}\right]$. It is obvious that the projection of the gravitational acceleration and earth rotation angular velocity in the navigation coordinate is $a_{n}^{b}=\left[\begin{array}{lll}0 & 0 & g\end{array}\right]$ and $w_{i e}^{n}=\left[\begin{array}{llll}w_{i e} \cos L & 0 & w_{i e} \sin L\end{array}\right]$. The transformation matrix is defined as follows [3]:

$$
C_{n}^{b}=\left[\begin{array}{ccc}
\cos \psi \cos \theta & \sin \psi \cos \theta & -\sin \theta \\
-\sin \psi \cos \phi+\cos \psi \sin \theta \sin \phi & \cos \psi \cos \phi+\sin \psi \sin \theta \sin \phi & \cos \theta \sin \phi \\
\sin \psi \sin \phi+s \cos \psi \sin \theta \cos \phi & -\cos \psi \sin \phi+\sin \psi \sin \theta \cos \phi & \cos \theta \cos \phi
\end{array}\right]
$$

Therefore equations can be written as $a^{b}=C_{n}^{b} a^{n}$, and $w_{i e}^{b}=C_{n}^{b} w_{i e}^{n}$. We can obtain the value of initial euler angles through the calculation. The pitch angle is $\theta=-\arcsin \left(a_{x}^{b} / g\right)$, the roll angle is $\phi=\arctan \left(a_{y}^{b} / a_{z}^{b}\right)$, and the Yaw angle is $\varphi=\arccos \left(\left(w_{i e} \sin L \sin \theta+w_{i b x}^{b}\right) /\left(w_{i e} \cos L \cos \theta\right)\right)$.

\subsection{Attitude Compensation}

Because the initial alignment process ignores the body shake and influence of the drift of the inertial sensor, $C_{n}^{b}$ must be revised [12].

(1) We use angular rate errors and acceleration errors from the EKF filter to compensate measurements of the angular rate and acceleration:

$$
\begin{aligned}
\hat{w}_{i b k}^{b} & =w_{i b k}^{b}-\delta w_{i b(k-1)}^{b} \\
\hat{a}_{i b k}^{b} & =a_{i b k}^{b}-\delta a_{i b(k-1)}^{b}
\end{aligned}
$$

(2) Updating quaternion. The uncompensated transformation quaternion from $\mathrm{k}$ to $\mathrm{k}-1$ in body frame is written as:

$$
q_{b(k, k-1)}^{b(k-1, k-1)}=\cos \frac{\|\Phi\|}{2}+\Phi \frac{\sin \frac{\|\Phi\|}{2}}{\|\Phi\|}
$$

where $\Phi$ denotes the equivalent rotation vector in the body coordinate from k to k-1. The uncompensated quaternion at $\mathrm{k}$ moment is:

$$
q_{b(k, k-1)}^{n}=q_{b(k-1, k-1)}^{n} \otimes q_{b(k, k-1)}^{b(k-1, k-1)}
$$

(3) We use errors of attitude angles to compensate the quaternion. Estimation of the quaternion at $\mathrm{k}$ moment can be expressed as:

$$
\begin{gathered}
q_{b(k, k)}^{n}=q_{b(k, k-1)}^{n} \otimes q_{b(k, k)}^{b(k, k-1)} \\
q_{b(k, k)}^{b(k, k-1)}=\cos \frac{\left\|-\delta \varphi_{k}\right\|}{2}-\delta \varphi_{k} \frac{\sin \frac{\left\|-\delta \varphi_{k}\right\|}{2}}{\left\|-\delta \varphi_{k}\right\|}
\end{gathered}
$$

\subsection{EKF Design}

Although errors between the measurement and real value in the navigation coordinate are rare, it is necessary to establish an angle errors model and estimate errors to gain the precise attitude through EKF. 
The state vector is defined as in EKF:

$$
\delta X=\left[\begin{array}{lllllllll}
\delta \varphi_{E} & \delta \varphi_{N} & \delta \varphi_{U} & \delta w_{i b x} & \delta w_{i b y} & \delta w_{i b z} & \delta a_{i b x} & \delta a_{i b y} & \delta a_{i b z}
\end{array}\right]
$$

where $\delta \varphi_{E}, \delta \varphi_{N}, \delta \varphi_{U}$ are errors of attitude angles of the pedestrian in the navigation coordinate. $\delta w_{i b x}, \delta w_{i b y}, \delta w_{i b z}$ are errors of angular rates of X, Y, Z axis in the inertial coordinate. $\delta a_{i b x}, \delta a_{i b y}, \delta a_{i b z}$ are errors of accelerations of $\mathrm{X}, \mathrm{Y}, \mathrm{Z}$ axis in the inertial coordinate.

State equation: $\delta X_{k, k-1}=\phi_{k} \delta X_{k-1, k-1}+W_{k-1}$

State transition matrix is:

$$
\phi_{k}=\left[\begin{array}{ccc}
I_{3 \times 3} & \Delta t C_{b(k+1, k)}^{n} & 0 \\
0 & I_{3 \times 3} & 0 \\
0 & 0 & I_{3 \times 3}
\end{array}\right]
$$

where $C_{b(k+1, k)}^{n}$ denotes the uncompensated attitude matrix at k moment, $\Delta t$ denotes the sampling period, $W_{k-1}$ denotes system noises.

Observation vector: $\delta Z_{k}=\left[\varphi_{k, k-1}-\varphi_{k} \quad w_{i b k}^{b}-C_{n(k, k-1)}^{b} w_{i e}^{n}\right]$

where $\varphi_{k+1, k}$ denotes the uncompensated yaw angle matrix at $\mathrm{k}$ moment, $\varphi_{k}$ denotes the yaw angle calculated from gyroscope measurements.

Observation equation: $Z_{k}=H X_{k-1, k-1}+V_{k-1}$

where $V_{k-1}$ denotes observation noises.

The observation transition matrix is:

$$
H=\left[\begin{array}{ccc}
0 & 0_{1 \times 4} & 0 \\
0_{4 \times 1} & I_{4 \times 4} & 0_{4 \times 1} \\
0 & 0_{1 \times 4} & 0
\end{array}\right]
$$

Estimating system states through the EKF equation and compensating errors by using a closed loop form are useful to ensure the accuracy of attitude angles and improve reliability of the digital platform.

\subsection{Fusion Algorithm}

In this system, we can obtain the optimal value by fusing information from GPS and INS. The state vector used in EKF is written as follows:

$$
X=\left[\begin{array}{llllll}
E & N & S & A & \varphi & L
\end{array}\right]
$$

where $\mathrm{E}, \mathrm{N}$ and A denote the eastern, northern and vertical position of pedestrians in the navigation frame, $\mathrm{S}$ and $\mathrm{L}$ denote speed and the step length of pedestrians, $\varphi$ denotes the yaw angle of pedestrians.

State equation is:

$$
\left\{\begin{array}{l}
E_{k+1}=E_{k}+L_{k} \cos \varphi_{k}+w_{E} \\
N_{k+1}=N_{k}+L_{k} \sin \varphi_{k}+w_{N} \\
S_{k+1}=S_{k}+w_{S} \\
A_{k+1}=A_{k}+w_{A} \\
\varphi_{k+1}=\varphi_{k}+w_{\varphi} \\
L_{k+1}=L_{k}+w_{L}
\end{array}\right.
$$


where dynamic noises of the position satisfy $w_{E} \sim N\left(0, \delta_{E}^{2}\right), w_{N} \sim N\left(0, \delta_{N}^{2}\right)$, $w_{A} \sim N\left(0, \delta_{A}^{2}\right)$, dynamic noises of the velocity, yaw angle, step length satisfy $w_{S} \sim N\left(0, \delta_{S}^{2}\right), w_{\varphi} \sim N\left(0, \delta_{\varphi}^{2}\right), w_{L} \sim N\left(0, \delta_{L}^{2}\right)$.

According to the derivation and analysis, the state-transition matrix is presented as follows:

$$
\phi_{k} \approx \frac{\partial f}{\partial X}=\left[\begin{array}{cccccc}
1 & 0 & 0 & 0 & L \cos \varphi & \sin \varphi \\
0 & 1 & 0 & 0 & -L \sin \varphi & \cos \varphi \\
0 & 0 & 1 & 0 & 0 & 0 \\
0 & 0 & 0 & 1 & 0 & 0 \\
0 & 0 & 0 & 0 & 1 & 0 \\
0 & 0 & 0 & 0 & 0 & 1
\end{array}\right]
$$

Measurements from GPS and barometer are used as observations for EKF. Two situations are considered according to the quality of GPS signals.

(1) The GPS signals are available with four satellites in view at least. The observation vector is written as:

$$
Z=\left[\begin{array}{llllll}
E_{G P S} & N_{G P S} & S_{G P S} & A_{I N S} & \varphi_{I N S} & L_{I N S}
\end{array}\right]
$$

where $E_{G P S}, N_{G P S}, S_{G P S}$ denote the eastern position, northern position and speed from GPS in the navigation coordinate. $A_{I N S}$ denotes the altitude from the barometer, $\varphi_{I N S}$ denotes the yaw angle after EKF, and $L_{I N S}$ denotes the step length derived from the model.

Observation equation is:

$$
\left\{\begin{array}{l}
E_{G P S}=E_{k}+w_{E G P S} \\
N_{G P S}=N_{k}+w_{N G P S} \\
S_{G P S}=S_{k}+w_{S G P S} \\
A_{I N S}=A_{k}+w_{A I N S} \\
\varphi_{I N S}=\varphi_{k}+w_{\varphi I N S} \\
L_{I N S}=L_{k}+w_{L I N S}
\end{array}\right.
$$

The Observation transition matrix is:

$$
H=\left[\begin{array}{c}
H_{G P S} \\
H_{I N S}
\end{array}\right]
$$

where $H_{G P S}=\left[\begin{array}{ll}I_{3 \times 3} & 0_{3 \times 3}\end{array}\right]$ denotes the observation matrix of GPS which connects the observed value from GPS with the state vector. $H_{I N S}=\left[\begin{array}{ll}0_{3 \times 3} & I_{3 \times 3}\end{array}\right]$ denotes the observation matrix of INS which connects the observed value from INS with the state vector.

The observation noise matrix is:

$$
P=\left[\begin{array}{cc}
P_{G P S} & 0 \\
0 & P_{I N S}
\end{array}\right]
$$

(2) The GPS signal is unavailable, and the observation vector is:

$$
Z=\left[\begin{array}{llll}
S_{I N S} & A_{I N S} & \varphi_{I N S} & L_{I N S}
\end{array}\right]
$$

where $S_{I N S}$ denotes the speed of the pedestrian from INS, $A_{I N S}$ denotes the altitude derived from the barometer, $\varphi_{I N S}$ denotes the yaw angle after correction, and $L_{I N S}$ denotes the step length based on the model.

Observation equation is: 


$$
\left\{\begin{array}{l}
S_{I N S}=S_{k}+w_{\text {SINS }} \\
A_{I N S}=A_{k}+w_{\text {AINS }} \\
\varphi_{I N S}=\varphi_{k}+w_{\varphi I N S} \\
L_{I N S}=L_{k}+w_{\text {LINS }}
\end{array}\right.
$$

The observation transition matrix: $H=\left[I_{4 \times 4}\right]$

\section{Experiment and Simulation}

In order to verify the effectiveness of the algorithm and assess the positioning accuracy of the system, we finish several typical experiments:

In the first test, we choose a regular graph under the condition of no GPS signal. Walking along the interior corridor edge, the shape likes a ' $\mathrm{T}$ '. The trajectory starts from zero, including eight 90-degree turns, which is shown in Fig. 4. We estimate the biggest errors in the eastern and northern direction and the position error after returning to the origin.

In the second test, we choose a casual graph when GPS signals are available. Walk optionally and go back to the origin which is marked. This trajectory includes straight lines, curves, walking backward, and so on, which is shown in Fig. 5. Results of both tests are listed in Table 2 .

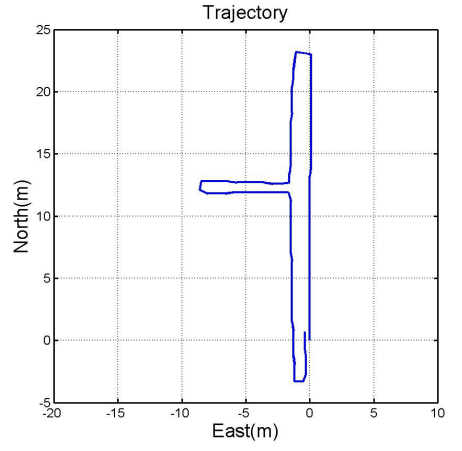

Figure 4: Trajectory in the First Test

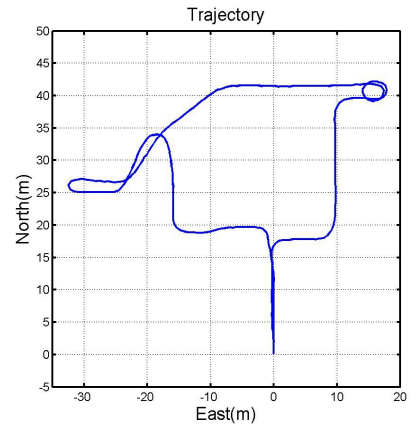

Figure 5: Trajectory in the Second Test

\begin{tabular}{|c|c|c|}
\hline Item & test1 & test2 \\
\hline Step number (count) & 100 & 272 \\
\hline Length in the eastern direction (m) & 9 & 50.4 \\
\hline Length in the northern direction (m) & 24 & 42.2 \\
\hline Biggest bias in the eastern direction (m) & 0.5 & $/$ \\
\hline Biggest bias in the northern direction (m) & 0.7 & 0.1 \\
\hline Errors when back to the origin (m) & 0.86 & $/$ \\
\hline
\end{tabular}

Table 2: Test Results

\section{Conclusion}

The gait navigation system integrates a six-axes sensor and a barometer, communicates with PC and mobile phones through bluetooth, and uses the lithium battery to supply power. At the same time, this system takes into account features of the pedestrian kinematics model that the measurement of the three-axes acceleration changes periodically when walking, which avoids the peak detection error and improves accuracy of the step detection by smooth filtering. Several corrected parameters are obtained after a large number of experiments. Establishing a 
nonlinear model about the step length ensures accuracy of the step length. After the initial alignment, the posture is corrected by the kalman filtering algorithm which is based on quaternion and an accurate digital platform is obtained. Then we apply different navigation strategies according to different environments by assessing superiority of GPS signals, and use the data fusion algorithm to ensure reliability, continuity of the positioning precision. The positioning accuracy of this navigation system can reach $3 \%$ through the experimental analysis. This system verifies accuracy and effectiveness of the algorithm, and realizes the superior accuracy of the indoor and outdoor navigation and positioning.

\section{References}

[1] Y.S. Suh, S. Park, Pedestrian Inertial Navigation with Gait Phase Detection Assisted Zero Velocity Updating[C]. The 4th International Conference on Autonomous Robots and Agents 2009 (ICARA), IEEE, Wellington, 336-341(2009)

[2] S. Moafipoor, Intelligent Personal Navigator Supported by Knowledge-Based Systems for Estimating Dead Reckoning Navigation Parameters[D]. USA: The Ohio State University(2009)

[3] M. Langer, S. Kiesel, C. Ascher, G.F. Trommer, Deeply Coupled GPS/INS Integration in Pedestrian Navigation Systems in Weak Signal Conditions[C]. 2012 International Conference on Indoor Positioning and Indoor Navigation, IEEE, Sydney, 1-7(2012)

[4] Y. Cui, K.B. Ariyur, Bounding Inertial Drift with Human Gait Dynamics for Personal Navigation [C]. 2011 International Systems Conference, IEEE, Montreal, 28-33(2011)

[5] H.M. Zhang, W.Z. Yuan, Q. Shen, T. Li, H.L. Chang, A Handheld Inertial Pedestrian Navigation System With Accurate Step Modes and Device Poses Recognition[J]. sensors journal. 15(3): 14211429(2014)

[6] L. Ojeda, J. Borenstein, Non-GPS Navigation for Security Personnel and First Responders[J]. Journal of Navigation. 60(3): 391-407(2007)

[7] T. Gadeke, J. Schmid, M. Zahnlecker, W. Stork, K.D. Muller-Glaser, Smartphone pedestrian navigation by foot-IMU sensor fusion[C]. 2012 Ubiquitous Positioning, Indoor Navigation, and Location Based Service (UPINLBS 2012), IEEE, Helsinki, 1-8(2012)

[8] D. Gusenbauer, C. Isert, J. krosche, Self-Contained Indoor Positioning on off-the-shelf Mobile Devices[C]. 2010 International Conference on Indoor Positioning and Indoor Navigation, IEEE, Zurich, 1-9(2010)

[9] Y. Li, J.J. Wang, A robust pedestrian navigation algorithm with low cost IMU[C]. 2012 International Conference on Indoor Positioning and Indoor Navigation, IEEE, Sydney, 1-7(2012)

[10] S. Asano, Y.Wakuda, N. Koshizuka, K. Sakamura. Pedestrian Dead-reckoning Unit for Navigation System using Mobile Device[C]. The 1st Global Conference on Consumer Electronic 2012(GCCE), IEEE, Tokyo, 530-534(2012)

[11] A.R. Jimenez, F. Seco, C. Prieto, J. Guevara, Indoor pedestrian navigation using an INS/EKF framework for yaw drift reduction and a foot-mounted IMU[C]. 7th Workshop on Positioning, Navigation and Communication, IEEE, Dresden, 135-143(2010)

[12] H. Yin, IMU Indoor pedestrian dead reckoning research based on foot-mounted[D]. China: Nanchang University(2013) (In Chinese) 